\title{
A counterexample to unique continuation in dimension two
}

\author{
Niculae MandaChe
}

\begin{abstract}
We construct a non-zero solution $u \in C_{0}^{\infty}(\mathbb{C})$ of the equation $\bar{\partial} u=$ $V u$ for a certain $V$ which belongs to $L^{p}$ for any $p<2$. The same is done in arbitrary dimension $d \geq 2$ for the Laplace equation with a first order term $\Delta v+W \cdot \nabla v=0$ and for the Dirac equation $\mathcal{D} w+W w=0$, with $W \in L^{p}$ for any $p<2$. The construction is based on a Weierstrass product in the unit ball. Although its poles accumulate at the boundary, it is flat at the boundary if we remove small disjoint discs around the poles.
\end{abstract}

\section{Introduction.}

Let $\Omega \subset \mathbb{R}^{n}$ be a connected open set. We say that an equation $P(x, \partial / \partial x) u=0$ has the unique continuation property in $\Omega$ if any solution $u$ which vanishes in a non-empty open subset vanishes identically. We are interested in the equation

$$
\bar{\partial} u=V u \quad \text { in } \mathbb{C}=\mathbb{R}^{2},
$$

where $\bar{\partial}$ is the Cauchy-Riemann operator, $\bar{\partial}=\frac{1}{2}\left(\partial / \partial x_{1}+i \partial / \partial x_{2}\right)$ and $V \in$ $L_{\text {loc }}^{1}(\mathbb{C})$. We will also consider here the Laplace equation with a first order term:

$$
\Delta v+W \cdot \nabla v=0
$$

and the Dirac equation:

$$
\mathcal{D} w+W w=0,
$$

with the (zero mass) Dirac operator $\mathcal{D}=\sum_{i=1}^{d} \alpha_{i} \partial / \partial x_{i}$. The $\alpha_{i}$ are matrices of order $m$ satisfying $\alpha_{i}^{*}=-\alpha_{i}$ and the Clifford commutation relations: $\alpha_{i} \alpha_{j}+\alpha_{j} \alpha_{i}=-2 \delta_{j k} . m$ can be chosen $2^{[d / 2]}$.

There is extensive literature on unique continuation and strong unique continuation properties (in the later, one supposes the solution to vanish 
to infinite order at a point instead of vanishing on an open set), see [6]. Although the problem of strong unique continuation has been almost settled, it is a long standing problem whether equations (1.1), (1.2) and (1.3) have the unique continuation property when the coefficients are $L_{\text {loc }}^{1}$. We give here a negative answer, constructing solution with compact support for (1.1),(1.2) and (1.3) with coefficients in $L^{p}$ for any $p<2$. This is optimal in dimension 2. The best positive result belongs to Wolff [5]: in dimension $d$, if $W \in L^{d}$ then (1.2) and (1.3) have the unique continuation property.

While this paper was awaiting publication, a new way of constructing counterexamples to unique continuation was found by Kenig and Nadirashvili [2]. Their result was improved and extended from dimension two to arbitrary dimension by Koch and Tataru [3].

We fix the function $F(t)=t^{2} \ln ^{-3}(t+2)$ on $[0, \infty)$. It has the property that $F(a t) \leq a^{2} F(t)$ for $a>1$ and $F(t) / \tilde{F}(t)$ is bounded, $\tilde{F}$ being the greatest convex function with $\tilde{F}(t) \leq F(t)$ for any $t \geq 0$. Therefore the set

$$
L^{F}\left(\mathbb{R}^{d}\right)=\left\{f: f \text { measurable on } \mathbb{R}^{d}, \int F(|f|) d x<\infty\right\}
$$

coincides with the Orlicz space associated with $\tilde{F}$ on $\left(\mathbb{R}^{d}, d x\right)$ (see e.g., [1]). It is a Banach space such that $L^{p} \subset L_{l o c}^{F}$ for $p<2$.

Theorem 1.1. There are $u, V$ which satisfy (1.1) such that $u \in C_{0}^{\infty}(\mathbb{C})$, $\operatorname{supp} u=B(0,1)$ and $V \in L^{F}(\mathbb{C})$. There are a real-valued [both $v$ and $W$ are real valued] $v \in C_{0}^{\infty}(\mathbb{C})$ and $W \in L^{F}(\mathbb{C})$ satisfying $(1.2)$, such that $\operatorname{supp} v=B(0,1)$.

Corollary 1.2. For any $d \geq 2$, there are non-zero smooth solutions $v$ realvalued and $w$, for the equations (1.3) and (1.2) respectively, with compact support in $\mathbb{R}^{d}$ and such that the coefficients $W$ belong to $L^{F}\left(\mathbb{R}^{d}\right)$.

Notations. $\quad C, C^{\prime}, C^{\prime \prime}$ will stand for absolute positive constants, not necessarily the same in different formulae. The constants which will be carried from a formula to another will be numbered $C_{1}, C_{2}, \ldots$. We write $f(x) \sim g(x)$ in $M$ if there are absolute constants $0<c<C$ such that $c f(x) \leq g(x) \leq C f(x)$ for all $x \in M$.

We use the variable $z=x_{1}+i x_{2}$, identifying $\mathbb{C}$ with $\mathbb{R}^{2}$. If $\beta \in \mathbb{N}^{2}$ is a multi-index, $\partial^{\beta}=\left(\partial / \partial x_{1}\right)^{\beta_{1}}\left(\partial / \partial x_{2}\right)^{\beta_{2}}$ and $|\beta|=\beta_{1}+\beta_{2} . B(a, r)$ is the ball of center $a$ and radius $r$ and $\stackrel{\circ}{B}(a, r)$ is its interior. If $x$ is a real number, we denote by $[x]$ the greatest integer in $(-\infty, x]$. 


\section{Proofs.}

We start by constructing a meromorphic function in the unit disk $B(0,1)$ as a Weierstrass product. It vanishes to infinite order at the boundary, in a sense which is made precise by Lemma 2.1 below:

$$
f(z)=\prod_{j=2}^{\infty} \frac{1}{1-\left(\frac{z}{1-1 / j}\right)^{j^{2}}}
$$

Let us denote the poles of $f$ by

$$
z_{k, l}=\frac{k-1}{k} \exp \left(2 \pi i l / k^{2}\right), \text { for } k \geq 2,0 \leq l<k^{2} .
$$

Then each of the sets

$$
M_{k, l}=\left\{\begin{array}{cc}
z: & |z| \in\left[\frac{2 k-3}{2 k-1}, \frac{2 k-1}{2 k+1}\right), \\
& \arg z \in\left[(2 l-1) \pi / k^{2},(2 l+1) \pi / k^{2}\right)
\end{array}\right\}, k \geq 2,0 \leq l \leq k^{2},
$$

contains the corresponding pole of $f$. Moreover, these sets form a partition of the annulus $\{z:|z| \in[1 / 3,1)\}$. We will also need slightly bigger sets:

$$
\tilde{M}_{k, l}=\left\{\begin{array}{c}
z: \\
\arg z \in\left[(4 l-3) \pi / 2 k^{2},(4 l+3) \pi / 2 k^{2}\right]
\end{array}\right\} .
$$

We have then

$$
\begin{array}{rcc}
M_{k, l}+B\left(0, k^{-2} / 8\right) & \subset \tilde{M}_{k, l} . \\
B\left(z_{k, l}, k^{-2} / 8\right) & \subset & M_{k, l} .
\end{array}
$$

Lemma 2.1. The infinite product (2.1) converges to a meromorphic function in the unit open disk, and it converges to 0 elsewhere. Let

$$
g_{k, l}(z)=f(z)\left(z-z_{k, l}\right)
$$

be the analytic function in $M_{k, l}$ obtained by multiplicatively removing the pole in this region. Then the following estimate holds:

$$
\left|g_{k, l}(z)\right| \sim k^{-2} \prod_{j=2}^{k-1}(1-1 / j)^{j^{2}}|z|^{-k^{3} / 3+k^{2} / 2} \leq \exp \left(-k^{2} / 6+2 k\right), \quad z \in \tilde{M}_{k, l} .
$$


Proof of Lemma 2.1. We divide the product (2.1) in three parts: the factors with index $j<k$, the factors with $j>k$ and the factor with $j=k$.

We estimate first the modulus of $\left(\frac{z}{1-1 / j}\right)^{j^{2}}$ for $z \in \tilde{M}_{k, l}$, for $2 \leq j \leq k-1$. Since $\frac{4 k-7}{4 k-3} \leq|z|$ we have $\frac{4 j-3}{4 j+1} \leq|z|$, so

$$
\begin{aligned}
& \left|\left(\frac{z}{1-1 / j}\right)^{j^{2}}\right| \geq\left(\frac{1-1 /(j+1 / 4)}{1-1 / j}\right)^{j^{2}} \rightarrow e^{1 / 4} \text { as } j \rightarrow \infty, \text { hence: } \\
& \left|\left(\frac{z}{1-1 / j}\right)^{j^{2}}\right| \geq C_{1}>1 .
\end{aligned}
$$

In the same way we obtain for $|z| \leq \frac{4 k-1}{4 k+3}$ :

$$
\left|\left(\frac{z}{1-1 / j}\right)^{j^{2}}\right| \leq C_{2}<1 \text { for } j \geq k+1 \text {. }
$$

Since $|\ln (|1 /(1-a)| \cdot|a|)|=|\ln (|1-1 / a|)| \leq C_{3}|1 / a|$ for $|a| \geq C_{1}>1$, putting $a=(z /(1-1 / j))^{j^{2}}$, we obtain from (2.5), using again $|z| \geq(4 k-7) /(4 k-3)$ as $z \in \tilde{M}_{k, l}$ :

$$
\begin{aligned}
|\ln | \frac{\prod_{j=2}^{k-1} \frac{1}{1-\left(\frac{z}{1-1 / j}\right)^{j^{2}}}}{\prod_{j=2}^{k-1}\left(\frac{1-1 / j}{z}\right)^{j^{2}}}|| & \leq C_{3} \sum_{j=2}^{k-1}\left|\frac{1-1 / j}{(4 k-7) /(4 k-3)}\right|^{j^{2}} \\
& \leq C_{3} \sum_{j=2}^{k-1}(1-1 / j)^{j^{2}}(1+1 /(k-7 / 4))^{j^{2}} \\
& \leq C_{3} \sum_{j=2}^{k-1} \exp -\frac{j(k-7 / 4-j)}{k-7 / 4} \\
& \leq C_{3} e^{3}+C_{3} \sum_{j=2}^{k-2} \exp (-\min (j, k-7 / 4-j) / 2) \\
& \leq C_{3} e^{3}+2 C_{3} \sum_{j=1}^{\infty} \exp (-j / 2+3 / 8)=C_{4} .
\end{aligned}
$$

Since $|z|^{k} \sim 1$ for $\frac{4 k-7}{4 k-3} \leq|z| \leq 1$, the above gives in this region:

$$
\left|\prod_{j=2}^{k-1} \frac{1}{1-\left(\frac{z}{1-1 / j}\right)^{j^{2}}}\right| \sim \prod_{j=2}^{k-1}\left|\frac{1-1 / j}{z}\right|^{j^{2}} \sim|z|^{-k^{3} / 3+k^{2} / 2} \prod_{j=2}^{k-1}(1-1 / j)^{j^{2}}
$$


We have the following upper bound for the right hand side above:

$$
\begin{aligned}
& |z|^{-k^{3} / 3+k^{2} / 2} \prod_{j=2}^{k-1}(1-1 / j)^{j^{2}} \\
& \leq\left(\frac{4 k-7}{4 k-3}\right)^{-k^{3} / 3+k^{2} / 2} \exp \sum_{j=2}^{k-1} j^{2}(-1 / j) \\
& \leq \exp \left(\left(-k^{3} / 3+k^{2} / 2\right) \frac{-1}{k-7 / 4}-\sum_{j=2}^{k-1} j\right) \\
& \leq \exp \left(k^{2} / 3+k-k(k-1) / 2+1\right) \\
& \leq \exp \left(-k^{2} / 6+2 k\right), \quad \text { for }|z| \geq \frac{4 k-7}{4 k-3} .
\end{aligned}
$$

This proves the inequality in (2.4). Since for (2.5) to hold we only need $|z| \geq \frac{4 k-7}{4 k-3}$, we infer from (2.7) and (2.8) that the product (2.1) converges to 0 if $|z| \geq 1$.

Now, for the factors with index greater than $k$ in the definition of $f$, we use that $|\ln (1-a)| \leq C_{5}|a|$ for $|a| \leq C_{2}<1$. Via the relation (2.6) this can be applied for $a=\left(\frac{z}{1-1 / j}\right)^{j^{2}}$ and we obtain for $|z| \leq \frac{4 k-1}{4 k+3}$ :

$$
\begin{aligned}
\sum_{j=k+1}^{\infty}\left|\ln \frac{1}{1-\left(\frac{z}{1-1 / j}\right)^{j^{2}}}\right| & \leq C_{5} \sum_{j=k+1}^{\infty}\left|\frac{1-\frac{1}{k+3 / 4}}{1-1 / j}\right|^{j^{2}} \\
& \leq C_{5} \sum_{j=k+1}^{\infty}\left(1-\frac{1}{k+1}\right)^{j^{2}}\left(1+\frac{1}{j-1}\right)^{j^{2}} \\
& \leq C_{5} \sum_{j=k+1}^{\infty} \exp \left(\frac{j^{2}}{j-1}-\frac{j^{2}}{k+1}\right) \\
& \leq C_{5} e^{3 / 2}+C_{5} \sum_{j=k+2}^{\infty} \exp (k+2-j)=C_{6} .
\end{aligned}
$$

Hence the product $\prod_{j=k+1}^{\infty} \frac{1}{1-\left(\frac{z}{1-1 / j}\right)^{j^{2}}}$ is uniformly absolutely convergent for $|z| \leq \frac{4 k-1}{4 k+3}$, and

$$
e^{-C_{6}} \leq\left|\prod_{j=k+1}^{\infty} \frac{1}{1-\left(\frac{z}{1-1 / j}\right)^{j^{2}}}\right| \leq e^{C_{6}} .
$$


This implies that the product $(2.1)$ is convergent everywhere in $\stackrel{\circ}{B}(0,1)$. Indeed, for $|z| \leq \frac{4 k-1}{4 k+3}$ the product up to the factor $k$ is a meromorphic function and the rest of the product is absolutely convergent.

For the middle factor with $j=k$ in (2.1), notice first that the logarithm is a quasi-isometry in the region $0<c \leq|z| \leq C$ and $-3 \pi / 4 \leq \arg z \leq$ $3 \pi / 4$. More generally, $\left|z-z^{\prime}\right| \sim\left|\ln \frac{z}{z^{\prime}}\right|$ for $|z|,\left|z^{\prime}\right| \in[c, C]$ and $\arg \frac{z}{z^{\prime}} \in$ $[-3 \pi / 4,3 \pi / 4]$. Since $z_{k, l}^{k^{2}}=(1-1 / k)^{k^{2}}$ we have:

$$
\left|\frac{1}{1-\left(\frac{z}{1-1 / k}\right)^{k^{2}}}\right| \sim \frac{1}{k^{2}\left|\ln \frac{z}{z_{k, l}}\right|} \sim \frac{1}{k^{2}} \frac{1}{\left|z-z_{k, l}\right|} \text { for } z \in \tilde{M}_{k, l} .
$$

It remains to multiply the relations $(2.7),(2.9)$ and $(2.10)$ to obtain the first part of (2.4).

Proof of Theorem 1.1. We choose a function $\phi \in C^{\infty}(\mathbb{C})$ such that:

$$
\phi(z)=\phi(|z|)= \begin{cases}1 & \text { for }|z| \geq 1 \\ |z|^{2} & \text { for }|z| \leq 1 / 2 .\end{cases}
$$

We require $\phi$ to be increasing on $[0, \infty)$ and $\phi(r) / r$ to have only one critical point in $(0, \infty)$ and that to be non-degenerate. For a choice of the quantities $\varepsilon_{k}$ to be performed later, under the constraint $0<\varepsilon_{k} \leq k^{-2} / 8$, we define our function solution of equation (1.1):

$$
u(z)=f(z) \prod_{k=2}^{\infty} \prod_{l=0}^{k^{2}-1} \phi\left(\frac{z-z_{k, l}}{\varepsilon_{k}}\right) .
$$

Notice first that the product is well defined, since by (2.3) the sets of points where each of the factors is different from 1 are disjoint. Since the poles $z_{k, l}$ of $f$ are simple and $\phi(z)=z \bar{z}$ in a neighborhood of $0, u$ is smooth in $\stackrel{\circ}{B}(0,1)$. For $z \in M_{k, l}$ we have

$$
\begin{aligned}
\bar{\partial} u(z)=\bar{\partial}\left(f(z) \phi\left(\frac{z-z_{k, l}}{\varepsilon_{k}}\right)\right) & =f(z) \bar{\partial} \phi\left(\frac{z-z_{k, l}}{\varepsilon_{k}}\right) \\
& =f(z) \frac{1}{\varepsilon_{k}}(\bar{\partial} \phi)\left(\frac{z-z_{k, l}}{\varepsilon_{k}}\right)
\end{aligned}
$$

and we obtain that the equation (1.1) is satisfied with a potential

$$
V=\sum_{k=2}^{\infty} \sum_{l=0}^{k^{2}-1} \frac{1}{\varepsilon_{k}}\left(\frac{\bar{\partial} \phi}{\phi}\right)\left(\frac{z-z_{k, l}}{\varepsilon_{k}}\right) .
$$


The function $\bar{\partial} \phi / \phi$ has modulus $|z|^{-1}$ for $|z| \leq 1 / 2$ and vanishes for $|z|>1$. Since $\phi$ does not vanish outside 0 , we have that $|z| \bar{\partial} \phi(z) / \phi(z)$ is bounded in $B(0,1)$. Then

$$
\begin{aligned}
\int F(|V|) d x_{1} d x_{2} & \leq C \sum_{k=2}^{\infty} \sum_{l=0}^{k^{2}-1} \int_{B\left(0, \varepsilon_{k}\right)}|z|^{-2} \ln ^{-3}\left(|z|^{-1}+2\right) d x_{1} d x_{2} \\
& \leq C \sum_{k=2}^{\infty} k^{2} 2 \pi \int_{0}^{\varepsilon_{k}}-x^{-1} \ln ^{-3} x d x \\
& \leq C^{\prime} \sum_{k=2}^{\infty} k^{2} \ln ^{-2} \varepsilon_{k} .
\end{aligned}
$$

We choose

$$
\varepsilon_{k}=\min \left(k^{-2} / 8, e^{-k^{1.7}}\right),
$$

and obtain that the series above is convergent, so $V \in L^{F}$. It remains to check that the solution (2.11) is smooth.

Take $\tilde{g}_{k, l}(z)=z^{k(k-1)(2 k-1) / 6} g_{k, l}(z)$. The relation (2.4) from Lemma 2.1 implies that $\left|\tilde{g}_{k, l}(z)\right| \sim\left|\tilde{g}_{k, l}\left(z_{k, l}\right)\right|$ for $z \in \tilde{M}_{k, l}$. From (2.2), each point $z \in M_{k, l}$ is the center of a ball of radius $k^{-2} / 8$ contained in $\tilde{M}_{k, l}$. Since $\tilde{g}_{k, l}$ is holomorphic in $\tilde{M}_{k, l}$, we can apply the Cauchy representation formula using as contour the boundary of this ball, to obtain $\left|\partial^{\alpha} \tilde{g}_{k, l}(z)\right| \leq C_{\alpha}\left(k^{2|\alpha|}\right)\left|\tilde{g}_{k, l}\left(z_{k, l}\right)\right|$ for $z \in M_{k, l}$. Then differentiating $g_{k, l}(z)=\tilde{g}_{k, l}(z) z^{-k(k-1)(2 k-1) / 6}$ we obtain for a suitable choice of the constants $C_{\alpha}^{\prime}$ :

$$
\left|\partial^{\alpha} g_{k, l}(z)\right| \leq C_{\alpha}^{\prime} k^{3|\alpha|}\left|g_{k, l}(z)\right| \text { for } z \in M_{k, l}
$$

Let us define

$$
\tilde{\phi}(z)=\frac{\phi(z)}{z}
$$

Since $\tilde{\phi}$ is a symbol of order -1 , all its derivatives are bounded. Then, with the notation of Lemma 2.1,u(z)= $\frac{g_{k, l}(z)}{\varepsilon_{k}} \tilde{\phi}\left(\frac{z-z_{k, l}}{\varepsilon_{k}}\right)$ for $z \in \tilde{M}_{k, l}$. Using (2.13), the definition of $\varepsilon_{k}$ and then (2.4) we obtain for $k$ big enough:

$$
\begin{aligned}
\left|\partial^{\alpha} u(z)\right| & \leq C_{\alpha}^{\prime \prime}\left(\max \left(\varepsilon_{k}^{-1}, k^{3}\right)\right)^{|\alpha|} \varepsilon_{k}^{-1}\left|g_{k, l}(z)\right| \\
& \leq C_{\alpha}^{\prime \prime} \exp \left((1+|\alpha|) k^{1.7}\right) C \exp \left(-k^{2} / 6+2 k\right), z \in M_{k, l}
\end{aligned}
$$


so all the derivatives of $u$ tend to zero as $|z| \rightarrow 1$, hence $u$ is smooth in $\mathbb{C}$.

For the second part of Theorem 1.1, we take $v=\operatorname{Re} u$, where $u$ is given by (2.11), but with one more condition on $\varepsilon_{k}$, which will change the choice (2.12) for finitely many values of $k$. In order to obtain a lower bound for $|\nabla v|$ we will need the following

Lemma 2.2. Let $h_{0}(z)=\operatorname{Re} \tilde{\phi}(z)$, where $\tilde{\phi}$ is given by (2.14). Then there are positive constants $c, C$ such that any real valued function $h \in$ $C^{2}(\stackrel{o}{B}(0,1))$, with $\left\|h-h_{0}\right\|_{C^{2}} \leq c$, has exactly two critical points $z_{1}, z_{2}$ and we have

$$
|\nabla h(z)| \geq C \min \left(\left|z-z_{1}\right|,\left|z-z_{2}\right|\right) .
$$

Proof. We have $h_{0}(z)=\frac{\phi(|z|)}{|z|} \cos (\arg z)$, so $\nabla h_{0}$ has two zeroes $\left(r_{\phi}, 0\right)$ and $\left(-r_{\phi}, 0\right)$, where $r_{\phi}$ is, by the choice of $\phi$, the unique zero of $\left(r^{-1} \phi(r)\right)^{\prime}$ in $(0, \infty)$ and $\left.\frac{d^{2}}{d r^{2}}\left(r^{-1} \phi(r)\right)\right|_{r=r_{\phi}} \neq 0$. Since $0, \pi$ are non-degenerate critical points of the function $\theta \rightarrow \cos \theta$, we can choose open sets $V_{1} \ni\left(r_{\phi}, 0\right)$ and $V_{2} \ni\left(-r_{\phi}, 0\right)$ such that $\nabla h_{0} \mid V_{i}$ is a diffeomorphism and the tangent mapping of its inverse is bounded. We still get these properties after replacing $h_{0}$ by $h$ if $\left\|h-h_{0}\right\|_{C^{2}} \leq c_{2}$ and $c_{2}$ is small enough. Taking $c_{2}$ even smaller, we can ensure that $h$ has exactly one critical point in each of $V_{1}, V_{2}$; let these be $z_{1}, z_{2}$. Then $|\nabla h|^{-1}\left|z-z_{i}\right|$ is uniformly bounded in $V_{i}$ with respect to different choices of $h$.

On the other hand, outside $V=V_{1} \cup V_{2}$ the gradient $\left|\nabla h_{0}\right|$ is bounded away from zero and so is $|\nabla h|$ if $\left\|h-h_{0}\right\|_{C^{1}} \leq c_{1}$ with $c_{1}=\frac{1}{2} \inf \{|\nabla h(z)|$ : $z \in B(0,1) \backslash V\}$. We choose then $c=\min \left(c_{2}, c_{1}\right)$.

Notice that Lemma 2.2 remains valid if we replace $\tilde{\phi}$ by $a \tilde{\phi}$, with $|a|=1$, since this is equivalent to composing $h_{0}$ with a rotation. This allows us to apply it to $h(z)=\left|g_{k, l}\left(z_{k, l}\right)\right|^{-1} \varepsilon_{k} v\left(z_{k, l}+\varepsilon_{k} z\right)$ which is a perturbation of $h_{0}=\operatorname{Re} \frac{g_{k, l}\left(z_{k, l}\right)}{\left|g_{k, l}\left(z_{k, l}\right)\right|} \tilde{\phi}$. From (2.13) we infer

$$
\frac{\left|\partial^{\alpha} g_{k, l}\left(z_{k, l}+\varepsilon_{k} z\right)\right|}{\left|g_{k, l}\left(z_{k, l}+\varepsilon_{k} z\right)\right|} \leq C_{\alpha}^{\prime}\left(\varepsilon_{k} k^{3}\right)^{|\alpha|}
$$

so making $\varepsilon_{k} k^{3}$ arbitrarily small we can make

$$
\left\|\frac{g_{k, l}\left(z_{k, l}+\varepsilon_{k} z\right)}{\left|g_{k, l}\left(z_{k, l}\right)\right|}-\frac{g_{k, l}\left(z_{k, l}\right)}{\left|g_{k, l}\left(z_{k, l}\right)\right|}\right\|_{C^{2}(B(0,1))}
$$


arbitrarily small. Indeed, if $\varepsilon_{k} k^{3} \leq C$ then (2.16) implies that $\left|g_{k, l}(z)\right| \sim$ $\left|g_{k, l}\left(z_{k, l}\right)\right|$ for $z \in B\left(z_{k, l}, \varepsilon_{k}\right)$, so we can replace the denominator in (2.16) with $\left|g_{k, l}\left(z_{k, l}\right)\right|$. Applying Lemma 2.2 we obtain that there is a constant $c_{1}>0$ such that for every $k$ and $l$, if $\varepsilon_{k} \leq c_{1} k^{-3}$ then there are two points $z_{k, l}^{(1)}, z_{k, l}^{(2)} \in B\left(z_{k, l}, \varepsilon_{k}\right)$ and $C>0$ such that for $z \in B\left(z_{k, l}, \varepsilon_{k}\right)$ we have:

$$
\left|g_{k, l}\left(z_{k, l}\right)\right|^{-1} \varepsilon_{k}|\nabla v(z)| \geq C \varepsilon_{k}^{-1} \min \left(\left|\frac{z-z_{k, l}^{(1)}}{\varepsilon_{k}}\right|,\left|\frac{z-z_{k, l}^{(2)}}{\varepsilon_{k}}\right|\right) .
$$

We take $\varepsilon_{k}=\min \left(k^{-2} / 8, c_{1} k^{-3}, e^{-k^{1.7}}\right)$ and $W(z)=-(\Delta v) \frac{\nabla v}{|\nabla v|^{2}}$. We use again that $\left|g_{k, l}(z)\right| \sim\left|g_{k, l}\left(z_{k, l}\right)\right|$ in $B\left(z_{k, l}, \varepsilon_{k}\right)$, to obtain in this ball from the first line of (2.15) and from (2.17):

$$
|W(z)|=\frac{|\Delta v(z)|}{|\nabla v(z)|} \leq \frac{\left(C_{(2,0)}^{\prime \prime}+C_{(0,2)}^{\prime \prime}\right) \varepsilon_{k}^{-3}\left|g_{k, l}(z)\right|}{C \varepsilon_{k}^{-3}\left|g_{k, l}\left(z_{k, l}\right)\right| \min _{i=1,2}\left|z-z_{k, l}^{(i)}\right|} \leq C^{\prime}\left(\frac{1}{\left|z-z_{k, l}^{(1)}\right|}+\frac{1}{\left|z-z_{k, l}^{(2)}\right|}\right)
$$

Since $v$ is harmonic in $M_{k, l} \backslash B\left(z_{k, l}, \varepsilon_{k}\right)$ we obtain that $W \in L^{F}$ :

$$
\begin{aligned}
\int F(|W|) d x_{1} d x_{2} & \leq C \sum_{k=2}^{\infty} \sum_{\substack{0 \leq l<k^{2} \\
j=1,2}} \int_{B\left(z_{k, l}^{(j)}, 2 \varepsilon_{k}\right)} F\left(\left|z-z_{k, l}^{(j)}\right|^{-1}\right) d x_{1} d x_{2} \\
& \leq C \sum_{k=2}^{\infty} 2 k^{2} 2 \pi \int_{0}^{2 \varepsilon_{k}}-x^{-1} \ln ^{-3} x d x \\
& \leq C^{\prime} \sum_{k=2}^{\infty} k^{2} \ln ^{-2} \varepsilon_{k}<\infty
\end{aligned}
$$

Proof of Corollary 1.2. The method is a standard one (see e.g., Theorem 2 in [4]): we wrap the solution around a compact submanifold to make it have compact support. Here the support will be a tube around a sphere of dimension $d-2$. We decompose $\mathbb{R}^{d}=\mathbb{R} \times \mathbb{R}^{d-1}$, and use the notation $x=\left(x_{1}, x^{\prime}\right)$ with $x^{\prime}=\left(x_{2}, \ldots x_{d}\right) \in \mathbb{R}^{d-1}$. Let $\left(W_{1}\left(x_{1}, x_{2}\right), W_{2}\left(x_{1}, x_{2}\right)\right)$ and $v$ be the coefficient of the gradient and the solution constructed in Theorem 1.1. Then in dimension $d \geq 3$ we take

$$
\begin{aligned}
\tilde{v}(x) & =v\left(x_{1},\left|x^{\prime}\right|-2\right) \\
\tilde{W}(x) & =\left(W_{1}\left(x_{1},\left|x^{\prime}\right|-2\right),\left(W_{2}\left(x_{1},\left|x^{\prime}\right|-2\right)-\frac{d-2}{\left|x^{\prime}\right|}\right) \frac{x^{\prime}}{\left|x^{\prime}\right|}\right)
\end{aligned}
$$


and using polar coordinates in $\mathbb{R}^{d-1}$ it is easy to check that (1.2) is satisfied.

For the equation (1.3), we take $w(x)=\mathcal{D} \tilde{v} \mathbf{v}_{\mathbf{0}}=\sum_{i=1}^{d} \partial_{i} \tilde{v}(x) \alpha_{i} \mathbf{v}_{\mathbf{0}}$, where $\mathbf{v}_{\mathbf{0}} \in \mathbb{C}^{m}$ is a fixed non-zero vector, and $W_{\mathcal{D}}=\frac{\bar{W} \cdot \nabla \tilde{v}}{|\nabla \tilde{v}|^{2}} \sum_{i=1}^{d} \partial_{i} \tilde{v} \alpha_{i}$, with the above $\tilde{v}, \tilde{W}$ (in dimension 2 we use $v, W$ provided by Theorem 1.1).

Remark. It is possible to obtain the above results by the standard procedure going back to Pliś (e.g., [4]). It consists of constructing a basic 'brick' of the solution and then gluing infinitely many of them. However, the closure under multiplication of the set of solutions of the $\bar{\partial}$ equation allowed to avoid this.

\section{References.}

[1] L.V. Kantorovich and G.P. Akilov, Functional analysis, Pergamon Press, Oxford, 1982.

[2] C. Kenig and N. Nadirashvili, A counterexample in unique continuation, preprint.

[3] H. Koch and D. Tataru, Sharp counterexamples in unique continuation for second order elliptic equations, submitted, preprint at http://www.math.berkeley.edu/ tataru/sucp.html.

[4] A. Pliś, A smooth linear elliptic differential equation without any solution in a sphere, Commun. Pure Appl. Math., 14 (1961), 599-617.

[5] T. Wolff, A property of measures in $\mathbb{R}^{n}$ and an application to unique continuation, Geometric and Functional Analysis, 2 (1992), 225-284.

[6] T. Wolff, Recent work on sharp estimates in second-order elliptic unique continuation problems, Journal of Geometric Analysis, 3 (1993), 621650 .

University of Bristol, School of Mathematics

UnIVERSITY WaLK, Bristol BS2 1TW, UK

E-mail address: Niculae.Mandache@bristol.ac.uk

On leave from:

Institute of Mathematics, Romanian ACADEmy

P.O. BoX 1-764, 70700 Bucharest, Romania

E-mail address: mandache@stoilow.imar.ro

RECEIVEd July 23, 1999. 